\title{
Tangence
}

\section{Problématique de l'enseignement des littératures francophones : compréhension et sympathie}

Frans C. Amelinckx

Numéro 49, décembre 1995

Les littératures francophones de l'Afrique et des Antilles

URI : https://id.erudit.org/iderudit/025877ar

DOI : https://doi.org/10.7202/025877ar

Aller au sommaire du numéro

Éditeur(s)

Tangence

ISSN

0226-9554 (imprimé)

1710-0305 (numérique)

Découvrir la revue

Citer cet article

Amelinckx, F. C. (1995). Problématique de l'enseignement des littératures

francophones : compréhension et sympathie. Tangence, (49), 49-62.

https://doi.org/10.7202/025877ar d'utilisation que vous pouvez consulter en ligne.

https://apropos.erudit.org/fr/usagers/politique-dutilisation/ 


\section{Problématique de l'enseignement des littératures francophones: compréhension et sympathie}

\section{Frans C. Amelinckx}

Avant d'aborder la problématique de l'enseignement des littératures francophones dans les programmes d'études de lettres au niveau supérieur, une réflexion générale sur la fonction de la littérature dans la formation me semble nécessaire. Dans un monde où la valeur dominante est utilitaire, comment peut-on justifier l'enseignement littéraire? Depuis de nombreuses années, les enseignants, les critiques débattent la question: la littérature véhicule de la culture générale? quelle littérature? celle consacrée? les classiques du monde occidental? Pour la littérature française, s'agit-il uniquement de transmettre à l'étudiant les ouvres matrices, c'est-à-dire consacrée par les manuels d'histoire littéraire et les programmes d'agrégation? Faut-il être d'accord avec Allan Bloom, ce critique acerbe de l'enseignement supérieur américain et de la culture générale en Amérique du Nord pour qui les lettres, tellement dévalorisées à l'université,

font penser au Marché aux puces de Paris, où au milieu de vieux déchets et de marchandises de pacotille on peut découvrir un trésor qui enrichira le chercheur astucieux. ${ }^{1}$

ou bien encore "Les lettres sont[-elles] donc devenues le dépôt, le caveau si l'on préfère de tous les classiques " 2 .

Pour Bloom, le débat concerne la lecture et la connaissance des textes classiques, fondamentaux, de la culture du monde occidental, gréco-romain et européen, qui doivent donner une réponse aux questions des jeunes.

Nous savons depuis Barthes que le choix du texte est déjà engagement:

1 Allan Bloom, L'âme désarmée. Essai sur le déclin de la culture générale, traduction de Paul Alexandre. Paris, Julliard/Guérin littérature, 1987, p. 317.

$2 \quad$ Ibid., p. 318 
50

L'écriture à laquelle je me confie est déjà tout institution; elle découvre mon passé et mon choix, elle me donne une histoire, elle affiche ma situation, elle m'engage sans que j'aie à le dire. ${ }^{3}$

Mon engagement personnel reprend l'affirmation de Térence "bomo sum; bumani nibil a me alienum punto", "je suis homme et rien de ce qui est humain de m'est étranger.. L'enseignement de la littérature en général et plus spécifiquement celle de la francophonie s'insère dans le cadre de la compréhension de l'autre en tant que personne humaine située dans le monde, c'est-à-dire que le discours est imbriqué dans une époque, une culture dans lesquels les éléments référentiels ont une part importante et reflètent une idéologie. Dans un texte, il y a tout un peuple, tout un pays, toute une culture qui font appel au lecteur. La fonction pédagogique est d'éveiller l'étudiant, le lecteur à l'image, au reflet de l'altérité. Cette fonction ne peut se réaliser que collectivement. Le rôle du professeur est de faciliter une recherche commune, la relation à autrui.

Bien que le concept d'altérité soit déconsidéré par certains critiques tels Henry Louis Gates et Bell Hooks pour qui l'altérité est un avatar du discours néocolonialiste ${ }^{4}$, elle permet néanmoins de reconnaitre l'être sous des aspects différents et de prendre conscience de l'humanité commune. Pour ce faire, j'aurai recours à un discours théorique, daté il est vrai, et entaché d'un élan bergsonien, celui de Paul Claudel développé dans son Art poétique dont les deux traités, "Connaissance du temps", et "Traité de la Co-naissance au monde et de soi-même" ouvrent des perspectives sur la compréhension de l'autre et sa relation intime avec le développement personnel. Le Traité, basé sur l'étymologie latine, met en évidence la notion des rapports entre connaître et naître, "notre être à chaque instant naît et co-naît aux autres corps dont il prend connaissance " 5 .

Rédigé au cours du deuxième séjour de Claudel en Chine, à Fou-tchéou en 1904, ce traité met en évidence une réflexion philo-

3 Roland Barthes, Le degré zéro de l'écriture, Paris, Seuil, 1953, p. 42.

4 Voir Henry Louis Gate, "Ethnic and Minorities Studies", Introduction to Scbolarship in Modern languages and Literatures, Joseph Gibaldi (dir.), New York, MLA 1992 et Bell Hooks, "Marginality as site of resistance", Out There: Marginalization and Contemporary Culture, Russell Ferguson (dir.), Martha Gever, Trinh T. Minh-ha, Cambridge, MIT Press, 1990.

5 Paul Claudel, Art poétique, Paris, Mercure de France, 1944 et Pierre Angers, Commentaire à l'art poétique, Paris, Mercure de France, 1949, p. 209-210. 
sophique pertinente non seulement à la connaissance du monde, mais plus précisément à un aspect de ce monde - la littérature, car "l'acte par lequel atteste la permanence des choses, par lequel, en dehors du temps, en dehors des circonstance et cause seconde, il formule l'ensemble des conditions permanentes dont la réunion donne à chaque chose son droit de devenir présente à l'esprit, lequel il la conçoit dans son cœur et répète l'ordre qu'il a créé, s'appelle la parole" ${ }^{6}$. Cette primauté de la parole dans le sens biblique d'acte créateur, d'acte d'écriture constitue un appel à l'existence, existence du texte, existence de l'auteur, existence du lecteur: "l'autre nous appelle à l'existence" ${ }^{7}$. Bien avant Mikhail Bakhtine, Claudel établit un rapport dialogique entre l'autre et le moi : "la clé d'un homme se trouve dans les autres: c'est le contact que nous avons avec le prochain qui nous éclaire sur nous-mêmes et d'où jaillit souvent la lumière sur notre caractère. C'est par la manière dont nous réagissons les uns à l'égard des autres que nous nous comprenons ou nous faisons comprendre ${ }^{8}$.

La notion de co-naître au texte, de le comprendre, c'est-à-dire de le faire sien, de se l'assimiler peut s'appliquer à l'expérience de la lecture et de la saisie d'un monde créé par la parole. Cependant, il ne faut pas se leurrer sur cette apparente simplicité, car la compréhension d'un texte ne se fait pas uniquement par la parole, chaque texte est inséré dans un contexte référentiel, historique, culturel et est dirigé par une intentionnalité qui esquisse une vision du monde. Cette intentionnalité, celle de l'auteur, est imbriquée dans son propre contexte littéraire. Ainsi la littérature francophone reflète une vision d'un monde, non pas unitaire, mais diversifié, enrichi par les apports d'autres cultures. Si de prime abord le lien unifiant les diverses littératures francophones est l'usage d'une langue, en l'occurrence le français, c'est aussi une langue qui déborde les limites imposées par le dictionnaire. Un auteur maghrébin, congolais, guadeloupéen inclut dans son texte les apports langagiers de sa culture. À l'intérieur d'une langue s'établissent les éléments de diversités, variétés dialectales ou régiolectales, vocables arabes, africains, créoles.

L'objectif de l'enseignement de la littérature francophone s'inscrit dans la pensée de la francophonie, celle d'un dialogue

$6 \quad$ Ibid., p. 343

7 Paul Claudel, Mémoires improvisées, Paris, Gallimard, 1954, p. 311.

8 Ibid. 
des cultures, d'un échange d'expériences personnelles et culturelles. À l'intérieur de la francophonie, il y a un refus de hiérarchie, ce qui ne se réalise pas nécessairement sans heurt ni conflit, mais qui résulte dans une démocratisation de la culture et dans la reconnaissance que chaque auteur peut exprimer et transmettre sa propre expérience et celle de son peuple.

Par leur nature même, les textes de la francophonie sont hétérogènes, mais l'enseignant et les lecteurs peuvent découvrir des points communs dans leur diversité. Tout texte riche.et complexe s'ouvre à une pluralité de significations et une pluralité de lectures. Il va sans dire qu'une analyse du discours pourrait relier plusieurs textes divers et faire ressortir les éléments sylistiques et rhétoriques unificateurs. L'élément unificateur pour moi se trouve dans le contexte humain, celui de la revendication à la dignité et à la valeur de l'être exprimée par les voix narratives.

Pour illustrer mon propos, je propose trois textes provenant des diverses régions francophones: Pluie et vent sur Télumée Miracle de Simone Schwarz-Bart situé aux Antilles ${ }^{9}$, Femmes d'Alger dans leur appartement d'Assia Djebar placé dans le contexte algérien de l'Islam ${ }^{10}$ et les méduses ou les orties de mer de Tchicaya U Tam'Si, qui a pour cadre le Congo ${ }^{11}$. Ces trois textes sont en effet profondément contestaires et subversifs car ils mettent en valeur le droit à la dignité de l'être. Chacun de ces textes, par ailleurs, illustre non seulement la diversité des sujets traités, mais aussi celle du contexte historique et social. De plus, chaque auteur a choisi une forme narrative mettant en valeur l'intentionnalité de son texte.

Pluie et vent sur Télumée Miracle se présente sous la forme d'un récit biographique par une narratrice à la première personne, Télumée elle-même. Le récit est aussi une quête du moi profond et de l'identité, "une narration du soi" ${ }^{12}$. Mais la narra-

9 Simone Schwarz-Bart, Pluie et vent sur Télumée Miracle, Paris, Seuil, 1972.

10 Assia Djebar, Femmes d'Alger dans leur appartement, Paris, Éditions des femmes, 1980.

11. Tchicaya U Tam'Si, Les méduses ou les orties de mer, Paris, Albin Michel, 1982.

12 Voir à ce propos Karen Smyley Wallace, "The Female and the Self in ScharzBart's Pluie et vent sur Télumée Miraclè, The French Review, vol. LIX, $\mathrm{n}^{\circ} 3$, 1986, p. 428-436; Kitzie McKinney, "Télumé's Miracle: The Language of the Other and the Composition of Self in Simone Schwarz-Bart's Pluie et vent sur 
trice/personnage principal est emblématique de toutes les femmes guadeloupéennes et son récit devient la chronique de la femme, chronique de l'exploitation à tous les niveaux, du plus élémentaire - l'exploitation sexuelle - à l'exploitation économique - celle des propriétaires Béké et celle de l'usine à sucre - et finalement à l'exploitation psychologique, celle de l'homme humilié qui se venge de sa situation sur la femme celle d'Elie et de l'ange Médard.

L'approche narrative de Simone Schwarz-Bart s'inscrit dans la tradition de l'oralité, celle du conteur/conteuse et, par ce fait, donne la voix, la parole à la femme, une parole qui lui avait été niée par la culture sociale, par la prédominance masculine - la femme n'étant qu'un corps, qu'un objet pour l'homme qu'il soit noir ou blanc. En décrivant sa vie, Télumée établit son identité, sa valeur en tant qu'être humain. Elle devient dans le récit la porteparole de toutes les femmes et plus précisément celle des femmes de sa propre famille, son aïeule Minerve, sa grand'mère Toussine "Reine sans Nom", sa mère Victoire. Télumée s'adresse directement au lecteur d'abord en donnant un contexte historique dans la première partie "Présentation des miens", contexte qui permet de situer les antécédents de Télumée et ceux de sa vie. Ce système narratif suggère une intimité entre lecteur/lectrice et narratrice. Par le simple "je" du texte, par la lecture, les souffrances, les peines, les joies, les échecs, les bonheurs sont ressentis non pas de l'extérieur, mais assimilés au moi profond du lecteur/lectrice. Cette technique renforce la prise de conscience du lecteur/lectrice et devient de ce fait une co-naissance au texte et à la vie de Télumée, vie fictive mais combien vraie, vie aussi de témoignages sur la quête de la dignité de soi et des autres, comme dans le cas de l'ange Médard quand les villageois expriment cette vérité à Télumée: "chère femme, l'ange Médard a vécu en chien et tu l'as fait mourir en homme [...] nous avons cherché un nom qui te convienne [...] Télumée Miracle [...]" 13 .

Par contre dans Femmes d'Alger, le système narratif, à l'exception d'une seule nouvelle - "Il n'y a pas d'exil" —, est celui de la narratrice omnisciente, choix d'un système narratif qui

Tëlumée Miracle", Modem Language Studies, vol. XIX, $\mathrm{n}^{\circ}$ 4, automne 1989 , p. 58-65; Clarisse Zimra, "Negritute in the Feminine Mode: The Case of Martinique and Guadeloupe", The Journal of Ethnic Studies, vol. XII, n" 1, 1984, p. 53-78.

Simone Schwarz-Bart, op. cit., p. 239. 
54

met en lumière l'importance de la parole et du regard, thèmes unificateurs des nouvelles et du titre emprunté au célèbre tableau de Delacroix. Ce thème du regard met en lumière la prise de possession de la femme objet non seulement par le regard du peintre violant le harem, mais aussi un regard démultiplié à l'infini par les spectateurs du tableau. Assia Djebar remarque dans sa postface que "le tableau lui-même est un regard volé "14 paradigme de tous les regards masculins qui dénudent ou qui dominent ${ }^{15}$. Comme celui des spectateurs du tableau, le regard de l'homme dans la tradition musulmane est un regard volé: «[...] on peut se rendre compte qu'à l'intérieur de cet Orient livré à lui-même l'image de la femme n'est pas perçue autrement [...] "16. Le regard omniscient de l'auteure n'en est plus un de domination, mais un révélateur de sympathie, celui d'une femme qui comprend et qui ressent elle-même la condition de ses soeurs situées dans le contexte culturel d'une tradition religieuse dominée par l'homme. Le recueil de nouvelles couvre une période historique, celle de la colonisation française et de la guerrre d'Algérie et celle de l'espoir vite avorté d'une reconnaissance de la dignité de la femme algérienne. La division en deux parties "Aujourd'hui " et "Hier" confère une profondeur temporelle. "Aujourd'hui" met en lumière les aspirations de la femme moderne dans une société en transition avec la nouvelle intitulée "Femmes d'Alger dans leur appartement", regard sur trois femmes, Sarah, femme moderne universitaire mariée à un médecin, Anne son amie française qui tente de se suicider, et la Fatma porteuse d'eau du hammam. La vie de la Fatma est un rappel du passé, mariée à treize ans, elle s'est enfuie dans l'espace et dans la misère afin d'échapper à son sort de femme opprimée. La deuxième nouvelle "La femme qui pleure" met en scène une femme maltraitée par son mari, qui se confie à un inconnu sur la plage, un prisonnier politique évadé. La nouvelle souligne une communauté de souffrance, un rapport entre deux opprimés au moyen de la parole, mais aussi par l'écoute: "Écouter l'autre! L'écouter simplement en le regardant [...] Vous parvient la voix de l'autre, de celui qui souffre ou qui a souffert [...] et se délivre " ${ }^{17}$. Par le biais de la douleur, la communication peut s'établir d'une manière éphémère, car la police

14 Assia Djebar, op. cit., p. 172.

15 Ibid., p. 174.

16 Ibid., p. 173.

17 Ibid., p. 74. 
vient arrêter l'inconnu et la nouvelle se termine sur l'échec de la communication, de la liberté et de l'espoir, car le seul recours est la pitié "la femme pleurait, la femme pleurait "18.

La deuxième partie, "Hier", est composée de quatre nouvelles dont la première, "Il n'y a pas d'exil", est la seule à la première personne. La puissance du "je" marque fortement la volonté de la femme d'être soi-même et non plus de dépendre des arrangements de mariage par la famille: "à vingt-cinq ans, après avoir été mariée, après avoir perdu successivement mes deux enfants, après avoir divorcé". Le "je" permet à la lectrice et à l'auteure de s'intégrer personnellement à la protagoniste/narratrice dans son refus d'accepter les conditions imposées par la tradition et la famille et de co-naître au texte, d'y participer pleinement. Les trois autres nouvelles reprennent la voix omnisciente et rattachent le passé à la situation contemporaine. Dans la nouvelle "Les morts parlent", Assia Djebar présente la femme forte, la grande dame qui, par sa noblesse et son comportement, transcende les restrictions de la tradition. "Jour de Ramadhan" rappelle les carêmes d'autant, mais aussi l'espoir brisé de la libération :" papoter, manger des gâteaux, s'empiffrer en attendant le lendemain, est-ce pour cela qu'il y a eu deuil et sang? Non, je ne l'admet pas... Moi... je croyais... que tout cela changerait, qu'autre chose viendrai, que....19. La dernière nouvelle, "La Nostalgie de la horde", est le récit de l'aieule, son mariage à douze ans, les mauvais traitements infligés par son mari, sa vie à l'arrivée des Français. La parole de l'aïeule est le rappel d'un passé distant puisqu'elle se rappelle de sa belle-mère lors de la conquête et la naissance d'une fille "tout juste bonne pour une race d'esclaves " 20 .

Par le biais de systèmes narratifs, Assia Djebar transforme le "le regard interdit" en regard lucide et contestataire, celui de la femme sur la situation de ses sœurs et le "son coupé", le silence imposé à la femme algérienne en parole vivante qui revendique la dignité de l'être pour la femme et refuse l'image créée par l'homme. Son texte invite les lecteur/lectrice à prendre conscience de la valeur de l'être et défait la "loi de l'invisibilité, la loi du silence " 21 . 
56

Dans Les méduses ou les orties de mer, roman central d'une triologie romanesque qui trace l'épopée de la famille Thom'Ndundie de 1910 à 1959, Tchicaya U Tam'Si explore les rapports entre les divers systèmes narratifs afin d'éclaircir le mystère de trois morts. On pourrait considérer ce texte comme une forme de roman policier. Le canevas au départ en est très simple, l'action étant basée sur deux morts accidentelles la dernière semaine de juin 1944, et une maladie comateuse. Il n'y a que trois personnages principaux, Kodia dit Elenga, mécanicien de locomotive sur le réseau C.F.C.O. (Chemin de Fer du CongoOcéan) Luambu-Lufwa Lumbu, commis aux écritures dans la Compagnie générale du Bas-Congo, Muendo Omar conducteur des travaux aux scieries réunies. Elenga est tué par les forces coloniales lors d'une grève de cheminots; Muendo meurt dans un accident à la scierie, et Luambu est trouvé dans le coma entre les tombes d'Elenga et de Muendo. Tous les trois sont liés par une solide amitié et leur mort et coma se situent exactement à un jour d'intervalle, le jeudi 22 juin pour Elenga, le vendredi 23 juin pour Muendo et le samedi 24 juin pour Luambu. La trame narrative est centrée sur une unité d'action, une unité de temps et une unité de lieu, toute l'action se situe à Pointe-Noire. Ainsi que le note Guy Daninos, le roman comporte en plus des unités classiques du théâtre un nombre d'éléments qui contribuent à sa théâtralité $^{22}$. Celle-ci met en rapport littérature et oralité et donc souligne la conscience de la communication faite à un auditoire ${ }^{23}$. Ainsi la première voix narrative, celle de l'auteur, se situe dans un prologue établissant l'importance de l'oral et des histoires fantastiques. Dans ce prologue, il s'agit d'une rumeur populaire au sujet d'un Blanc qui parcourait la ville indigène de Pointe-Noire et transformait hommes, femmes, enfants et chien en corned-beef, ce qui explique le refus d'achat de boîtes de conserve de la part des indigènes. Ce prologue, formule rituelle d'ouverture, a deux

22 Guy Daninos, "Les Méduses „, Présence africaine, $\mathrm{n}^{\circ} 125,1^{\text {er }}$ trimestre 1983, p. 255.

23 Thomas Melone souligne la théâtralité du discours romanesque africain dans un article traitant les problèmes du langage dans "Critique littéraire et problèmes du langage", Présence africaine, ${ }^{\circ} 731970$, p. 5. Cependant Alioune Tine ["Pour une théorie de la littérature africaine écrite", Présence africane, nos 133-134, 1985, p. 104] indique qu'il s'agit d'une oralité feinte, une transposition dans la fiction littéraire des modèles discursifs spécifiques à la tradition orale africaine. 
fonctions : attirer l'attention du public et inviter l'auditoire à participer à un monde différent ${ }^{24}$.

Ensuite l'auteur-narrateur situe le contexte de l'histoire: "Deux hommes sont morts dans la dernière semaine de juin 1944. Ils étaient trois à mourir [...] mais le troisième ne mourut pas, par miracle peut-être "25. Suit immédiatement une série de questions: "Qui peut savoir? Comment, pourquoi? Où? Quelle coïncidence, dirait-on? Quelle évidence niait-on?" ${ }^{26}$ Le récit fonctionne par rapport aux questions et élucide petit à petit le mystère par une serie de discours oppositionnels, Tchicaya U Tam'Si multiplie les plans narratifs, s'effaçant et réapparaissant à travers les interventions d'auteur et donnant la parole à tous les personnages secondaires par le biais de digressions, de dialogues, de souvenirs, de passages du réel au fantastique. "Quand on raconte ce que l'on rapporte, chacun ajoute une part d'invraisemblance pour faire vrai. 27 est la phrase clef qui amorce la multiplicité des signes narratifs. Le récit passe ensuite à un autre narrateur, André Sola, un chef de bureau évolué dans la Compagnie générale du BasCongo. Celui-ci se sentait concerné par le mystère de toutes ces morts et du coma: "Les fils embrouillés de toutes ces morts, André ne s'y trompait pas: malheur à lui, s'il ne se mettait pas en peine de les dénouer" 28. Pour ce faire, il va consulter un devin. Celui-ci véritable homme-récit, permet au romancier de passer à une nouvelle modalité discursive, celle de la divination qui informe l'action et relie le passé au présent. Donc le devin va révéler les antécédents, l'enfance de Kodia Elenga, ensuite le devin passe à la vie de Luambu-Lufwa Lumbu qui est cousue d'énigmes, mais de nouveau, la divination n'agit plus pour les événements récents. L'obscurité reste, le devin l'explique par ces mots: "Ça c'est le domaine de la mort, n'y entre pas vivant qui veut $" 29$.

Pour le dernier protagoniste, Muendo, André Sola établit son passé, ses origines à travers le discours de la communauté. La

24 Alioune Tine, "Pour une théorie de la littérature africaine écrite", Présence africaine, $\mathrm{n}^{\mathrm{os}} 133-1341985$, p. 103.

25 Tchicaya U Tam'Si, op. cit., p. 13.

26 Ibid., p. 13-14.

27 Ibid., p. 26.

28 Ibid., p. 31.

29 Ibid., p. 62. 
58

modalité du discours est celle de l'opinion commune, une série de dialogues brefs en discours direct sans préciser les locuteurs. Justement dans ce discours, le lecteur apprend par des témoins oculaires la rencontre de Luambu, Elenga et Muendo et le commencement de leur amitié. Le discours de l'opinion commune est central, un récit enchâssé, car il décrit les circonstances de la rencontre lorsqu'un fou prédicateur provoque une bagarre entre les pêcheurs et les trois protagonistes. C'est de ce même récit que le roman tire son titre, car le fou avait prédit qu'il n'y aurait que des méduses dans les filets de pêche.

Ainsi que dans ses autres roman, Les cancrelats et Les phalènes, Tchicaya U Tam'Si centre sur une image principale la thématique du roman. Les méduses ou les orties de mer jouent un rôle fondamental qui intègre les diverses narrations, relie la multiplicité des signes et confère au roman son unité. En fait, la méduse avec ses multiples tentacules, sa forme amorphe, suggère que, sous la multiplicité des signes narratifs, il y a la présence d'une réalité floue - la mort de deux protagonistess dans la violence d'une grève ou dans un accident de travail, le coma du troisième - que l'on tente de comprendre et qui glisse pour le narrateur principal dans le fantastique. Les méduses, soit comme titre, soit comme image, deviennent une métaphore de la problématique du récit et, même, de la problématique de la vie. En filigramme, les méduses créent les points forts du récit: la rencontre des trois protagonistes, la mort d'Elenga et le coma de Luambu. Tchicaya U Tam'Si fait attention à la tradition africaine du devin, du signe de la réalité cachée, troublante derrière la réalité du quotidien. Son récit s'enracine dans les mythes et légendes contés le soir. Ainsi le prophète-fou, cause de la rencontre et de l'amitié de Elenga, de Muendo et de Luambu, est présent sur le marché, il réapparaît à plusieurs reprises, jouant à vendre des méduses, mais sous la folie, le lecteur prend concience de la présence de la prophétie, comme sous la vie on sent la présence de la mort: "Il sortit les méduses de l'eau, les posa par terre cérémonieusement. Il fit trois parts. Il dit: "Ils étaient trois", puis il se ravisa et en fit deux parts... "Ils étaient deux puis..." Il se ravisa à nouveau: "Non, le tout lui revient. Il est le seul qui reste de ceux qui m'ont sauvé la vie des mains des pêcheurs." Je vais sauver la tienne. "30 Puis le fou prophète se met à danser autour de la cuvette aux 
méduses, mais il fait un faux pas et tombe, le visage dans la cuvette. Muambu voit "les yeux révulsés, gluants et jaunâtres... une main aux doigts semblables à des serres d'oiseau de proie", qui essaient de le saisir et lui écorchent le visage et aussitôt il a une vision de la mort de ses amis. La conclusion reprend les mêmes gestes car Luambu dans le cimetière fait aussi un faux pas, perd l'équilibre et tombe: "Sa tête tomba sur quelque chose qui était mou, une terre fraîchement retournée qui avait un peu plus de consistance qu'un tas de méduses, qui, bien qu'un peu acide, ne donnait pas d'urticaire...."31.

Ainsi l'image centrale acquiert une signification à plusieurs niveaux. Il ne s'agit pas simplement de la Méduse de l'antiquité ni, comme le suggère Guy Daninos dans son compte rendu, "tout ce qui est de nature à neutraliser l'homme " 33 mais, à partir d'un élément concret, le romancier glisse de la réalité du quotidien soit à la réalité du fantastique, soit à la réalité socio-économique, passe d'une narration linéaire à une narration polyphonique, et donne une épaisseur temporelle à son récit en créant un déséquilibre spatiotemporel.

Le récit se situe dans la réalité de la violence quotidienne d'un pays colonisé, c'est-à-dire dans un contexte socio-économique. Les protagonistes travaillent tous pour des entreprises de colons. Les échos de la guerre européenne sont présents :

C'est la guerre. C'est le front partout. On aimerait plutôt être au front là-bas avec de Gaulle. Il faut être citoyen français pour cela, pour marcher au combat avec de Gaulle, sinon c'est derrière avec les Mboulou Mboulou - pas de casque avec la croix de Lorraine mais une chéchia rouge. ${ }^{34}$

Elenga est tué par la milice noire lorsqu'il participe à une grève de cheminots revendiquant de meilleures conditions de travail. Muendo, lui, est tué dans un accident de travail - la grande scie qui n'a qu'un panneau et une ligne tirée au sol comme mesure de sécurité. Muambu, bien qu'éduqué, un artiste calligraphe, un évolué, est venu à Pointe Noire à cause de difficultés avec son patron blanc. Cependant ces mêmes événements ne

31 Ibid., p. 265.

32 Guy Daninos, Présence africaine, $\mathrm{n}^{\text {os }} 3-4$, juillet-décembre, 1984, p. 259.

33. Tchicaya U Tam'Si, op. cit., p. 162.

34 Ibid., p. 162. 
60

sont pas interprétés selon une perspective socio-économique, ces mêmes événements sont perçus à travers une optique magique, mélange de tradition africaine et de religion catholique, celle d'André Sola, ancien séminariste et chef de bureau, qui possède dans sa vie le sens du mystère - mystère de la vie, mystère de la foi. Par conséquent, c'est lui qui colporte l'histoire que Muambu est un mauvais génie qui avait pris le corps d'un homme et qui était responsable de la mort de Muendo et Elenga. Cette perspective ouvre la narration à la dimension du fantastique.

La technique narrative est agencée comme la méduse. Au centre, l'ombrelle, l'événement capital, puis des séquences narratives itératives, souvent parallèles - récit d'André Sola, consultation de devin, dialogues multiples d'un auditoire, rêves, souvenirs, visions délirantes. Ces modalités polyphoniques permettent au lecteur de s'approcher de la réalité romanesque et de participer en fait au récit lui-même.

Grâce aux modalités du rêve, des souvenirs, de l'hallucination en particulier dans le cas de Muambu, le lecteur prend conscience des dimensions spatiotemporelles, du passé des protagonistes, de l'interaction des personnages, Noirs et Blancs. Ces modalités spatiales et temporelles agissent comme des points de réalité dans un système narratif qui joue, d'une part sur la réalité socioculturelle et économique, un point précis, Pointe-Noire en 1944, et une réalité fantastique - Luambu est-il un revenant, voit-il sa femme et son fils qui sont morts depuis des années, peut-on se protéger du mauvais sort? Toutes les modalités narratives participent à une mise en œuvre du récit.

Tchicaya U Tam'Si emploie aussi avec succès la technique narrative du nouveau roman, en particulier celle, fréquente dans les romans de Robbe Grillet, des récits parallèles dans lesquels un ou deux détails sont changés et le récit est repris dans une autre perspective.

Finalement, Tchicaya U Tam'Si a créé un récit qui, comme la méduse, donne l'urticaire, suscite le besoin absolu de continuer la lecture du récit non seulement par un jeu d'alternance narrative mais par son sujet même - l'interprétation de la réalité, la rupture de la solidité de la vie - et l'angoisse qui en résulte.

Cependant, il ne s'agit pas d'un roman négro-africain simplement traditionnel ou culturel. La méduse est plus qu'un signifié de la structure narrative ou de l'angoisse devant le mystère de la 
mort. Les méduses ou les orties de mer sont polyvalentes. Le récit est solidement ancré dans un espace temporel bien défini, dans un contexte socio-économique historique. Dans les dialogues de l'opinion publique, une phase est significative: "Le hasard, le hasard! le hasard n'explique rien du tout " ${ }^{35}$. La méduse, aux tentacules urticants, gluants, multiples, qui empêche une bonne pêche, c'est aussi l'argent. Le fou-prophète harangue la foule à propos de la convoitise, de la puissance de l'argent. Elenga est tué par les forces répressives de la Compagnie des Chemins de fer du Bas-Congo pour une question de salaire, Muendo meurt en préparant des planches pour le cercueil d'Elenga, rivé à son travail - un travail qui est une "vie tronçonnée [...] un interminable déchirement de la bille d'acajou " ${ }^{36}$. Luambu, lui, a eu des problèmes avec son patron à propos de faux dans les livres de comptabilité, il a des besoins constants d'argent pour entretenir la famille de ses parents. Au cours d'un départ précipité, Julienne, sa femme, et son enfant se noient. "L'argent fait mourir" annonce le fou, et ceci est repris par Luambu. L'homme s'enfonce dans cette masse gluante aux multiples tentacules qui détruit l'humanité.

Le roman rejoint ainsi le récit liminaire - le boycott de la viande en conserve. La mort n'est plus mystère mais révolte, refus de l'exploitation, refus de la magie et donc découverte du vrai mal - non plus celui d'André Sola mais celui contemporain de Luambu.

Tchicaya U Tam'Si, en intégrant une multiplicité de discours narratifs dans un seul récit, cerne une réalité qui n'est plus mystérieuse, ni magique, mais concrète, sociale, et politique. En tant que tel, Les méduses entre bien dans le cadre du roman africain comme le postule Mohamadou Kane: "le roman africain traduit largement la postulation d'un autre monde, de liberté, de progrès, il ne suppose pas de rupture avec le passé, l'africanité" 37 . De même, l'auteur s'intègre dans la pensée de la francophonie car "les valeurs qu'il nous lègue sont celles qui sous-tendent la tolérance, la générosité, le respect de l'autre "28.

35 Ibid., p. 87.

36 Ibid., p. 211, 213.

37 Mohamadou Kane, "Les paradoxes du roman africain", Présence africaine $\mathrm{n}^{\mathrm{O}} 139,1986$, p. 86 .

38 Arlette Chenoin-Degrange, "L'héritage de Tchicaya U Tam'Si", Europe, $\mathrm{n}^{\circ} 750$, octobre 1991, p. 140. 
62

Les trois textes choisis, hétérogènes par leur diversité d'espace, de temporalité, de sujet, sont néanmoins reliés par une intentionnalité de valeur humaine et sont un appel à la compréhension. Ils suggèrent une ouverture vers l'altérité des cultures et réduisent l'écart différentiel entre le lecteur et le texte, entre le lecteur et le monde représenté par l'écriture. L'enseignement des textes de la francophonie est un engagement humaniste, une conaissance à l'autre, et une sympathie intellectuelle. Ce genre de lecture évite le piège du discours occidental qui se veut totalisant et s'approprie le texte et l'expérience comme de simples matériaux culturels, historiques, psychologiques prêts à employer au lieu de découvrir une autre manière de voir le monde et de le comprendre, c'est-à-dire de co-naître à l'autre et à son expérience.

L'enjeu de l'enseignement des littératures francophones est d'harmoniser les tensions entre les cultures et de transmettre la réconciliation de l'être avec soi et les autres, c'est-à-dire d'être principe de vie et non pas le dépôt, le caveau des lettres, de donner une ouverture à l'autre et d'entrer en relation avec le texte et son message. Edouard Glissant suggère ce principe fondamental d'enseignement: "toute identité s'étend dans un rapport avec l'autre 39 .

39 Édouart Glissant, Poétique de la relation, Paris, Gallimard, 1990, p. 25. 